\title{
Provocative Testing with Intra-arterial Lidocaine during Bronchial Artery Embolization: An Underutilized Tool to Avoid a Devastating Complication
}

\author{
Joel E. Perchik ${ }^{1} \quad$ Jordan D. Perchik ${ }^{2}$
}

\author{
1Department of Interventional Radiology, Baptist Memorial \\ Hospital, Memphis, Tennessee, United States \\ ${ }^{2}$ Department of Radiology, University of Alabama, Birmingham, \\ Alabama, United States
}

J Clin Interv Radiol ISVIR 2019;3:199-200

You never want to learn about a technique for the first time from an attorney. The first time I heard of provocative testing in bronchial artery embolization (BAE) was from a colleague who was being sued by a patient who had suffered the devastating, but well-documented, complication of paraplegia due to spinal cord infarction. The physician was in deposition when the plaintiff's attorney asked if he had tried injecting lidocaine into the bronchial artery before embolization, which would provoke a temporary paralysis and suggest that there is a communication with the anterior spinal artery. This technique is essentially unknown within interventional radiology, but provocative testing has both patient outcome and medicolegal implications, referenced in malpractice lawsuits in cases of paraplegia and permanent neurological dysfunction.

Bronchial artery embolization is the most effective treatment for massive hemoptysis. The procedure is generally well tolerated and has success rates significantly higher than alternative treatments; however, it is not entirely risk free and one of the most feared adverse events of BAE is spinal cord infarction. The artery of Adamkiewicz typically has its origin off the aorta between T9 and T12, but in up to $5 \%$ of the population, the anterior spinal artery and right bronchial artery can share a common origin ( - Fig. 1). Serial angiography, the use of embolic particles greater than $350 \mu \mathrm{m}$, and superselective catheterization have been incorporated into the BAE technique to minimize the risk of anterior spinal cord infarction, but spinal cord infarction complications have not been eliminated entirely. ${ }^{1,2}$ Case reports of paraplegia after BAE continue to be reported and retrospective review of BAE cases report an incidence of neurologic complications, generally self-limited weakness and paresthesias, in 4 to $6 \%$ of cases. ${ }^{3}$

The provocative testing technique as we know it today was first described in 1986 by Doppman et al (then called the "spinal Wada test") who described the effects of lidocaine and phenobarbital injected directly into the artery of
Address for correspondence Jordan Perchik, MD, Department of Radiology, University of Alabama, 619 19th Street South, JT N338, Birmingham, AL 35249, United States (e-mail: jperchik@uabmc.edu).

Adamkiewicz on rhesus monkeys. ${ }^{4}$ When unable to identify the anterior spinal artery on angiography, the authors propose that infusion of phenobarbital or lidocaine could provoke transient paraplegia and suggest anastomotic connection to the anterior spinal artery. Specific recommendations regarding medication, dose, and details of the technique were not made, but results seemed promising.

Since being described in 1986, no further large scale studies have been performed to evaluate the spinal Wada test. In our institutional review board exempt review of provocative testing in BAE, we found that the technique is not well known and is inconsistently mentioned even in review articles on BAE or bronchial artery anatomy. Provocative testing is more commonly used by neurointerventional radiology

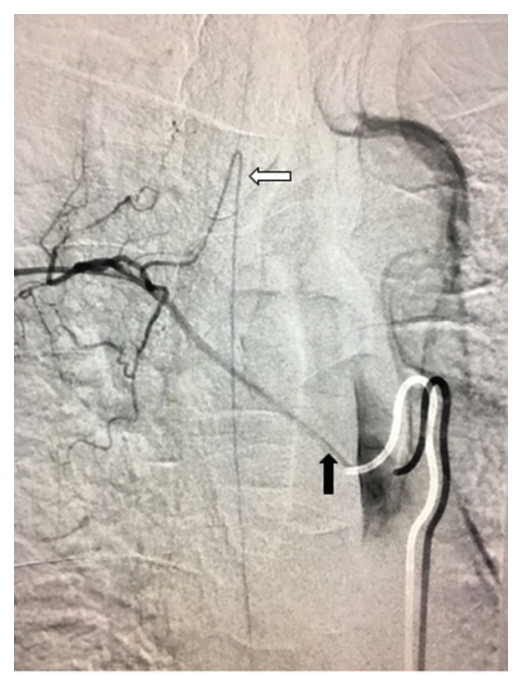

Fig. 1 Selective angiogram of the right bronchial artery (black arrow) with anastomosis to the artery of Adamkiewicz with the typical "hairpin loop" appearance (white arrow).
DOI https://doi.org/ $10.1055 / \mathrm{s}-0039-3401308$ ISSN 2457-0214.
License terms

$(\circledast) \Theta \circledast$ 
and neurosurgery in the treatment of cerebral and spinal arteriovenous malformations. The technique has a high negative predictive value and has a similar application in ruling out the risk of spinal cord infarction in BAE; proceeding with embolization in the setting of positive provocative testing has a high risk of spinal cord ischemia or infarction. Current practices suggest appropriate positioning of the microcatheter and injecting between 2.5 and $10 \mathrm{mg}$ of preservative-free $1 \%$ lidocaine after super selective catheterization. With spinal artery communication, the lidocaine should cause a temporary lower extremity weakness or paralysis. The catheter can then be repositioned and provocative testing repeated to assess for continued communication with the spinal artery. If the catheter cannot be repositioned beyond the spinal artery anastomosis or sufficiently out of the range of reflux, the physician and patient should be aware that there is a possibility of spinal cord infarction with embolization, and the decision can be made to proceed or abort the procedure. As described in the radiology literature, the technique requires the patient to communicate the symptoms during the procedure, but the use of somatosensory and motor evoked potentials has been suggested as an objective means of gauging neurologic response. ${ }^{5}$

Awareness and appropriate use of provocative testing in BAE will have a positive impact on patient safety. Questions remain regarding optimal medication dose, gauging patient response, adequate patient selection, best practices, and patient outcomes when employing the technique. Further research and refinement of the technique is necessary, but although it is not well established, provocative testing in BAE is a safe, effective, and reversible method of assessing risk of spinal cord infarction.

\section{Funding}

None.

\section{IRB Exempt}

No human or animal subjects or patient information were used in this project. The project is IRB exempt.

\section{Conflicts of Interest}

None.

\section{References}

1 Brown AC, Ray CE. Anterior spinal cord infarction following bronchial artery embolization. Semin Intervent Radiol 2012;29(3):241-244

2 Maramattom BV, Krishna Prasad BP, Padmanabhan S, Baby J. Spinal cord infarction after bronchial artery embolization. Ann Indian Acad Neurol 2016;19(1):156-157

3 Fruchter O, Schneer S, Rusanov V, Belenky A, Kramer MR. Bronchial artery embolization for massive hemoptysis: long-term follow-up. Asian Cardiovasc Thorac Ann 2015;23(1):55-60

4 Doppman JL, Girton M, Oldfield EH. Spinal Wada test. Radiology 1986;161(2):319-321

5 Davis MC, Deveikis JP, Harrigan MR. Clinical presentation, imaging, and management of complications due to neurointerventional procedures. Semin Intervent Radiol 2015;32(2):98-107 